\title{
Method of Measurement of Capacitance and Dielectric Loss Factor Using Artificial Neural Networks
}

\author{
Jerzy Roj, Adam Cichy \\ ${ }^{1}$ Institute of Measurement Science, Electronics and Control, Silesian University of Technology, Gliwice, Poland, \\ jerzy.roj@polsl.pl, adam.cichy@polsl.pl
}

\begin{abstract}
A novel method of dielectric loss factor measuring has been described. It is based on a quasi-balanced method for the capacitance measurement. These AC circuits allow to measure only one component of the impedance. However, after analyzing a quasi-balanced circuit's processing equation, it is possible to derive a novel method of dielectric loss factor measuring. Dielectric loss factor can be calculated after detuning the circuit from its quasi-equilibrium state. There are two possible ways of measuring the dielectric loss factor. In the first, the quasi-balancing of the circuit is necessary. However, it is possible to measure capacitance of an object under test. In the second method, the capacitance cannot be measured. Use of an artificial neural network minimizes errors of the loss factor determining. Simulations showed that the appropriate choice of the range of the detuning can minimize errors as well.
\end{abstract}

Keywords: Dielectric loss factor, quasi-balanced circuits, artificial neural network.

\section{INTRODUCTION}

$\mathrm{M}$ ANY PHYSICAL objects, which are dielectrics, can be in a steady state modeled as a combination of RC elements. Resistance $R$ models the energy loss (socalled active energy) and capacitance $C$ models the energy storage (so-called reactive energy). Such an object is, for example, electrical insulation. An important parameter of this model is the relationship between passive and active energy of the tested object. This relationship is called the dielectric loss factor. The phasor analysis of the object under test also shows the name of $\tan \delta$ because the loss factor is the tangent of the phase angle, by which the phase shift between current and voltage of the object is different from the $\pi / 2$ angle.

Dielectric loss factor is often measured in diagnostics of electrical insulation. It is an indicator of insulation moisture and allows the assessment of the degradation of the insulation.

There are many methods of measuring the dielectric loss factor used in practice. These are laboratory methods, such as the calorimetric method, alternating current bridges (e.g., Schering bridge), algorithmic methods and other.

The measuring method is shown below, which, after the modification and application of artificial neural network, allows the measurement of dielectric loss factor.

\section{MEASURING CIRCUIT}

The measuring circuit shown in Fig.1. [1] is a representative of a specific group of measuring circuits, the so-called quasi-balanced circuits [2-10]. These circuits are intended to measure AC impedance components. Their special feature is to have a selected state, the so-called quasi-equilibrium state.

Frequently, as in the circuit from Fig.1., this is the orthogonality of the two selected signals. The circuit is brought to this state by changes of a regulatory element. In the circuit from Fig.1. it can be an adjustable gain of the amplifier A or an adjustable conversion coefficient of the current/voltage converter B.

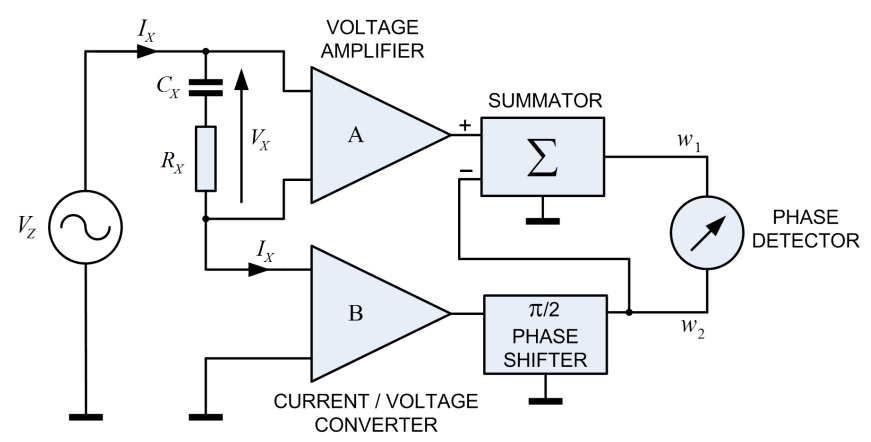

Fig.1. Quasi-balanced circuit for capacitance measuring of an object of RC type.

The measuring signals of the circuit from Fig.1., taking into account that the phase shifter $\pi / 2$ performs a multiplication by the imaginary unit $\mathrm{j}$, can be described as follows:

$$
w_{1}=A \cdot V_{X}-B \cdot \mathrm{j} I_{X}
$$

and

$$
w_{2}=B \cdot \mathrm{j} I_{X}
$$

As already mentioned, the circuit from Fig.1. has a state of quasi-equilibrium which means orthogonality of $w_{1}$ and $w_{2}$. This quasi-equilibrium state can be described by the equation:

$$
\operatorname{Re} \frac{w_{1}}{w_{2}}=0
$$

Because 


$$
\frac{w_{1}}{w_{2}}=\frac{A \cdot V_{X}-B \cdot \mathrm{j} I_{X}}{B \cdot \mathrm{j} I_{X}}
$$

and

$$
Z_{X}=\frac{V_{X}}{I_{X}}
$$

so (3) can be written as follows:

$$
\operatorname{Re} \frac{w_{1}}{w_{2}}=\frac{A}{B} \operatorname{Im} Z_{X}-1
$$

The equation determining the reactance of the serial object under test is given as follows:

$$
\operatorname{Im} Z_{X}=\frac{B_{0}}{A_{0}}
$$

where $A_{0}$ and $B_{0}$ are the voltage gain of the amplifier $\mathrm{A}$ and the conversion rate of the current/voltage converter in the quasi-equilibrium state, respectively.

Based on the reactance, the capacitance can be calculated for object under test as follows:

$$
C_{X}=\frac{A_{0}}{\omega B_{0}} .
$$

As already mentioned, an adjustable gain of the amplifier A or an adjustable conversion coefficient of the current/voltage converter $\mathrm{B}$ can be used as a regulatory element. More often an adjustable gain $A$ is used, due to simple calculations of the capacitance $C_{X}$.

\section{IDEA OF LOSS FACTOR MEASURING}

The circuit from Fig.1. is suitable only for measuring of a single impedance component. However, analysis of the circuit shown below allows the use of such circuit to measure the dielectric loss factor.

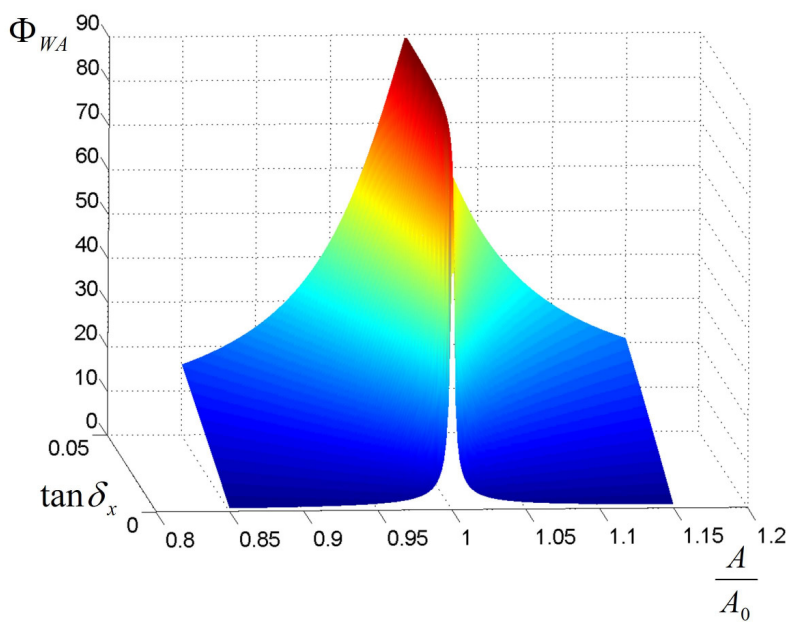

Fig.2. Illustration of (9).
Output signal under detection $\Phi_{W A}$ is in this circuit the phase shift angle between the $w_{1}$ and $w_{2}$. It can be described by the following equation [1]:

$$
\Phi_{W A}=\operatorname{arccot}\left(\frac{1}{\tan \delta_{X}} \cdot \frac{1-\frac{A}{A_{0}}}{\frac{A}{A_{0}}}\right) .
$$

This signal is a function of the control element A setting. It also depends on constants: dielectric loss factor $\tan \delta_{X}$ and setting of the regulatory element in quasi-equilibrium state $A_{0}$. Fig.2. is a graph showing the (9).

Dielectric loss factor $\tan \delta_{X}$ can be determined on the basis of (9), if the setting of the regulatory element is known, the corresponding value of the phase angle $\Phi_{W A}$ and gain of the voltage amplifier in quasi-equilibrium state $A_{0}$ is

$$
\tan \delta_{X}=\cot \Phi_{W A} \cdot\left(\frac{A_{0}}{A}-1\right) .
$$

In this case, we can also determine capacitance $C_{X}$.

If the gain of the voltage amplifier in quasi-equilibrium state $A_{0}$ is not known, two gain settings of the amplifiers $A_{1}$ and $A_{2}$ should be performed consecutively and corresponding values of phase angles $\Phi_{W 1}$ and $\Phi_{W 2}$ are readable:

$$
\Phi_{W 1}=\operatorname{arccot}\left(\frac{1}{\tan \delta_{\mathrm{x}}} \cdot \frac{1-\frac{A_{1}}{A_{0}}}{\frac{A_{1}}{A_{0}}}\right)
$$

and

$$
\Phi_{W 2}=\operatorname{arccot}\left(\frac{1}{\tan \delta_{\mathrm{x}}} \cdot \frac{1-\frac{A_{2}}{A_{0}}}{\frac{A_{2}}{A_{0}}}\right) .
$$

Dielectric loss factor can then be determined from the relationship:

$$
\tan \delta_{\mathrm{x}}=\frac{A_{2}-A_{1}}{A_{1} \cot \Phi_{W 1}-A_{2} \cot \Phi_{W 2}} .
$$

In this case, capacitance $C_{X}$ cannot be calculated.

Next we will discuss the method described by (10). This method allows the simultaneous measurement of capacitance (as the quasi-balanced circuit, after achieving the quasi-equilibrium state) and dielectric loss factor (after detuning from the quasi-equilibrium state).

In practice, the method of determining the value of dielectric loss factor using (10) is very sensitive to measurement errors of the phase angle and the relationship of $A / A_{0}$. Therefore, we attempted to determine the value of $\tan \delta_{X}$, using the artificial neural networks (ANN), because of their good approximation and generalization properties [11-13], [18]. 


\section{SiMULATION}

The study has been performed as a simulation, using the library Neural Network Toolbox, available in the Matlab environment [16]. The network with one hidden layer had been implemented and then it was learned by the Levenberg - Marquardt algorithm [17]. The sigmoid activation function of neurons in the hidden layer [14], [15] and a linear function in the output layer were used. The ANN structure is shown in Fig.3.

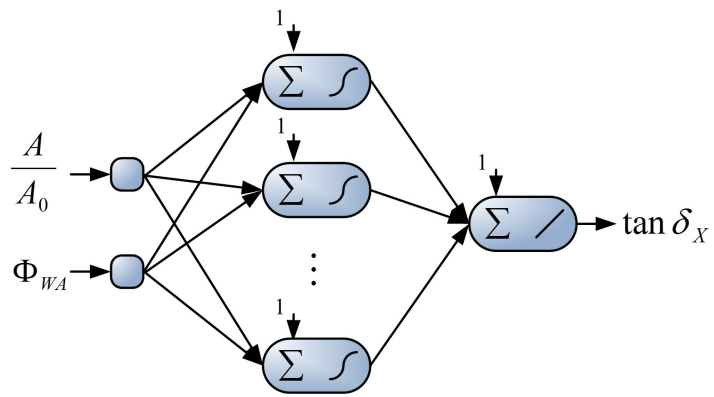

Fig.3. The structure of the ANN used for determining the dielectric loss factor.

A learning data set of 2500 items, i.e. 50 values of the $\Phi_{W A}$ and 50 values of the $A / A_{0}$ was given to the inputs of the ANN. The $2500 \tan \delta_{X}$ values were given on its output. The learning data set was generated using (9).

After the learning process, testing of the network with 40000 test element set was completed. Their values were obtained in a manner similar to the learning data set.

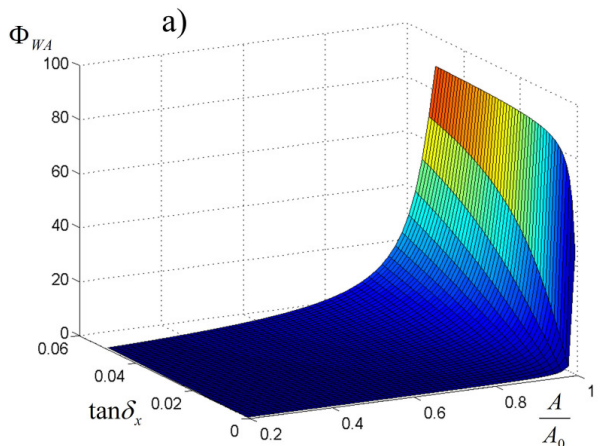

b)

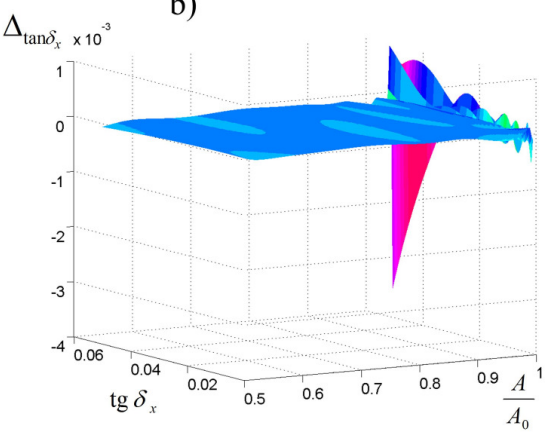

Fig.4. a) Values of $\Phi_{W A}$ vs. $A / A_{0}$ for the range of variation [0.200..0.999]) and $\tan \delta_{X}$ (for the range of variation $[0.001 \ldots 0.050]) ; b)$ surface of absolute error of neural determining the value of $\tan \delta_{X}$.
Preliminary studies have shown that the ANN with 12 neurons in the hidden layer (structure: 2-12-1) sufficiently approximates the (9). Increasing the number of neurons in the hidden layer and increasing the number of layers did not result in a significant improvement in approximation. Examples of the results of the relationship $\Phi_{W A}$ vs. $A / A_{0}$ (for the range of variation [0.200...0.999]) and $\tan \delta_{X}$ (for the range of variation [0.001...0.050]) are shown in Fig.4.

Fig.4.b) shows the strong increase in errors for $A / A_{0}$ for the range of variation $[0.200 \ldots 0.999]$ ) and $\tan \delta_{X}$ (for the range of variation $[0.001 \ldots 0.050])$; close to a value of 1 . This means the need for a significant detuning of the state of quasi-equilibrium to measure the dielectric loss factor. Therefore, the ANN learning data were limited to the range $[0.200 \ldots 0.800]$. The results obtained in the testing process are shown in Fig.5.

Fig.5.b) shows a graph of absolute error of dielectric loss factor calculation after changing the range of detuning of the $A / A_{0}$ parameter.

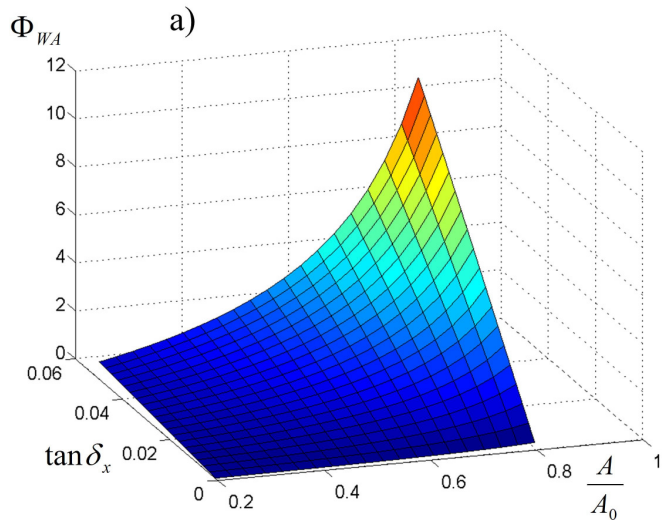

b)

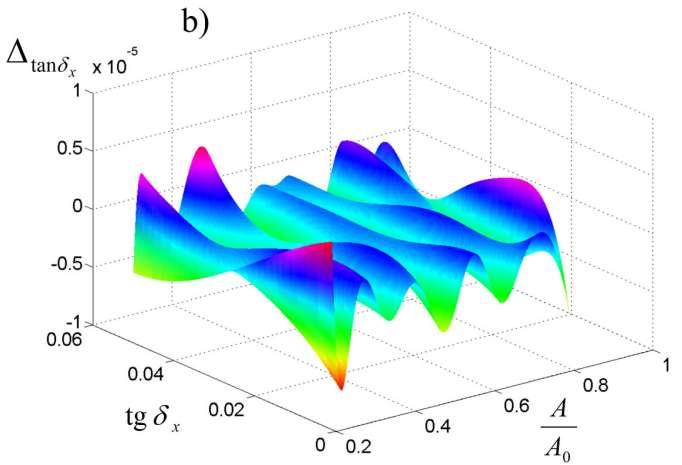

Fig.5. a) Values of $\Phi_{W A}$ vs. $A / A_{0}$ for the range of variation $[0.200 \ldots 0.800]$ ) and $\tan \delta_{X}$ (for the range of variation $[0.001 \ldots 0.050])$; b) surface of absolute error of neural determining the value of $\tan \delta_{X}$

It can be seen (Fig.5.b)) that the errors of neural determination of $\tan \delta_{X}$ (the assumed range of variation) do not exceed $0.5 \cdot 10^{-5}$. The expression of the calculation inaccuracies using the uncertainty requires, in the general case, implementation of the histogram of the errors and application of the method described in [19]. In situations where the shape of the histogram is similar to the standard 
error probability density function (normal, uniform, etc.), known statistical methods can be used.

However, note that the errors shown in Fig.5.b) relate to an ideal situation, i.e. that the $\Phi_{W A}$ and $A / A_{0}$ values resulting from (9) are given to the inputs of the neural network in the testing process.

In practice, the $\Phi_{W A}$ and $A / A_{0}$ values are measurement results, which are always obtained with a limited accuracy. In order to model a situation of this kind, tests were carried out for two cases. At first it was assumed that the $A / A_{0}$ and $\Phi_{W A}$ values have errors arising from 12 and 8-bit quantization, respectively. The assumption of such values is justified by the typical measurement chain [19]. In the second case it was assumed that both values are quantized with low, 6-bit resolution.

The quantization process implemented by the $\mathrm{A} / \mathrm{D}$ converter was modeled using dependence:

$$
n_{q}(x)=\operatorname{INT}\left(\frac{x}{q}+0.5\right)
$$

where $x$ and $n_{\mathrm{q}}(x)$ are quantities in the input and output of the $\mathrm{A} / \mathrm{D}$ converter, respectively, $q$ is the quantum value, $\operatorname{INT}(\bullet)$ is a function which assigns the integer part of its argument.
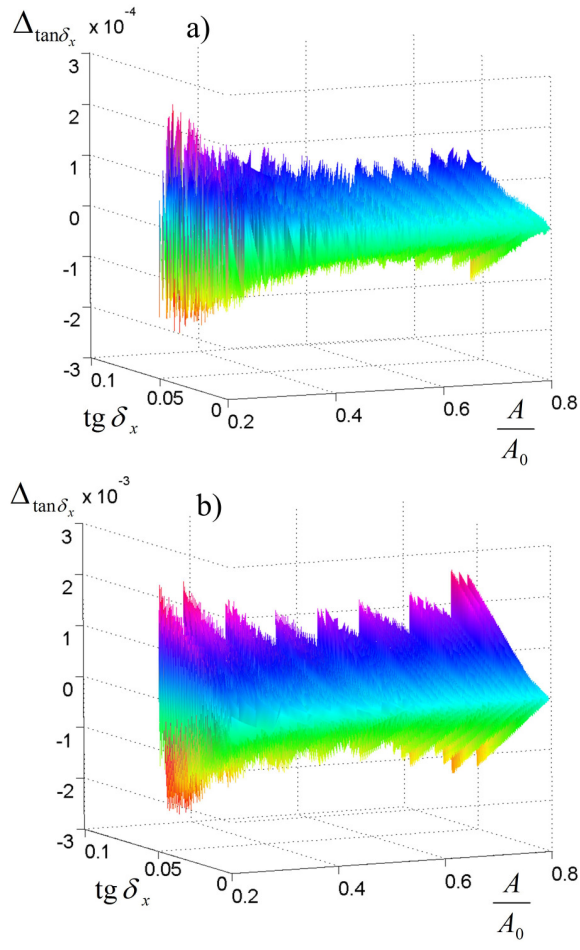

Fig.6. Surface of absolute error of neural determining of $\tan \delta_{X}$ for the $A / A_{0}$ and $\Phi_{W A}$ values quantized with a) 12-bit and 8-bit resolution, respectively; b) 6-bit resolution both.

After performing a multiplication:

$$
\widetilde{x}=n_{q}(x) \cdot q,
$$

relationship:

$$
x=\tilde{x}+\Delta_{q}
$$

is obtained, wherein $\Delta_{q}$ is a quantization error with uniform distribution in the interval $[-q / 2, q / 2]$. The results of these tests are shown in Fig.6.

It may be noted that the errors of neural determination of $\tan \delta_{X}$ are the smallest if the $A / A_{0}$ ratio is detuned to approximately 0.5 and it decreases with decreasing the value of $\tan \delta_{X}$. Histograms of $\tan \delta_{X}$ calculation errors for both cases are shown in Fig.7.
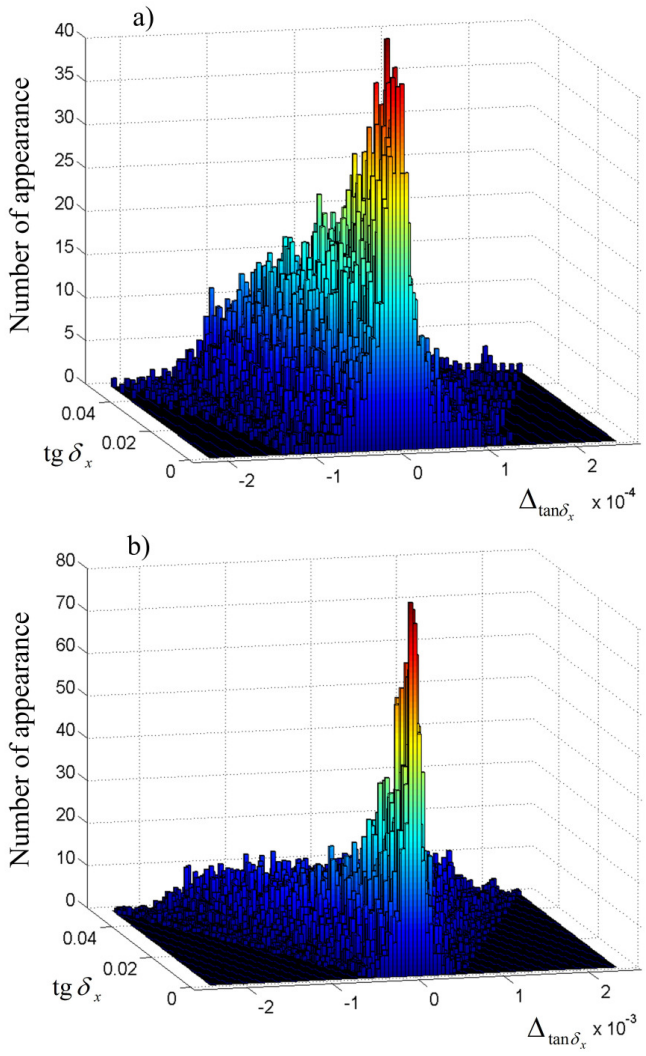

Fig.7. Error histograms of neural $\tan \delta_{X}$ calculation for the case of a) Fig.6a; b) Fig.6.b).

Error histograms of neural $\tan \delta_{X}$ calculation confirm previous observations. Namely, for small values of $\tan \delta_{X}$ the histogram shape is similar to a Gaussian distribution with a relatively small variance. With the $\tan \delta_{X}$ increase increases also the variance, while the shape of the histogram is changed to resemble uniform distribution, as can be seen especially in Fig.6.b).

\section{CONCLUSION}

The presented method of measuring of capacitance and dielectric loss factor is based on a circuit implementing the quasi-balanced method.

The capacitance of the object under test can be determined knowing the values of control elements in the quasiequilibrium state. When detuning from the quasiequilibrium state, the dielectric loss factor can be determined from (10). This eliminates one of the major 
disadvantages of the quasi-balanced circuits, namely, the ability to measure only one component of the impedance.

Calculation of the dielectric loss factor according to (10) can be charged with large errors. For this reason, the ANN technique has been used. Results of conducted researches indicate that a relatively simple feed-forward neural network with 12 neurons in the hidden layer approximates (10) sufficiently well. Approximation errors are in the range $0.5 \cdot 10^{-5}$. A situation in which the ANN input data have errors resulting from the quantization process was also modeled. Such a case is often encountered in practice, because the measurement results have mostly digital form, so they are processed by the A/D converter.

The results, which are illustrated in Fig.6. and Fig.7., allow formulating the following conclusions. Namely, the errors of $\tan \delta_{X}$ neural determination are smallest for the parameter $A / A_{0}$ equal to approximately 0.5 . They also decrease when values of $\tan \delta_{X}$ decrease. The histograms of errors presented in Fig.7. also show that the distribution of error probability density function has a shape similar to a Gaussian distribution with variance, the value of which increases with increasing $\tan \delta_{X}$. In situations where ANN input data $A / A_{0}$ and $\Phi_{W A}$ are burdened with significant errors arising from the low-resolution measurement chain, histogram of $\tan \delta_{X}$ errors (e.g., for $\tan \delta_{X}>0.04$ ) takes the form of uniform distribution. This means that in this case the inaccuracy of $\tan \delta_{X}$ neural determination is dominated by the errors resulting from the quantization process of $A / A_{0}$ and $\Phi_{W A}$. In conclusion, the use of ANN to determine $\tan \delta_{X}$ can be useful in practice, when the data needed to calculate it are burdened with errors.

\section{REFERENCES}

[1] Cichy, A., Skorkowski, A., Barwinek, S. (2013). Automated quasi-balancing in virtual quasi-balanced circuit designed to capacitance measurements. In 19th Symposium IMEKO TC 4 Symposium and 17th IWADC Workshop, 18-19 July 2013, Barcelona, Spain, 275-280.

[2] Skorkowski, A., Cichy, A. (2009). Virtual capacitance meter based on impedance modulus measurement. In XIX IMEKO World Congress, 6-11 September 2009, Lisbon, Portugal, 648-651.

[3] Amira, H., Hfaiedh, M., Valentin, M. (2009). Quasibalanced bridge method for the measurements of the impedances. IET Science Measurement \& Technology, 3 (6), 403-409.

[4] Atmanand, M.A., Jagadeesh Kumar, V., Murti, V.G.K. (1996). A microcontroller based quasi-balanced bridge for the measurement of L, C and R. IEEE Transactions on Instrumentation \& Measurement, 45 (3), 1-5.

[5] Burbelo, M.I. (2001). Universal quasi-balanced bridges for measuring the parameters of four-element two-terminal networks. Measurement Techniques, 44 (11), 1130-1133.
[6] Atmanand, M.A., Jagadeesh Kumar, V. (1996). A microcontroller based LCR meter. Microprocessors \& Micro-Systems, 20, 297-301.

[7] Atmanand, M.A., Jagadeesh Kumar, V., Murti, V.G.K. (1995). A novel method of measurement of L and C. IEEE Transactions on Instrumentation \& Measurement, 44 (4), 898-903.

[8] Marcuta, C., Fosalau, C., Petrescu, C. (2005). A virtual impedance measuring instruments based on quasi-balanced bridge. In 14th International Symposium on New Technologies in Measurement and 10th Workshop on ADC Modelling and Testing, 12-18 September 2005, Gdynia Maritime University, Poland, Vol. III, 517-523.

[9] Jagadeesh Kumar, V., Sankaran, P., Sudhakar Rao, K. (2003). Measurement of $C$ and $\tan \delta$ of a capacitor employing PSDs and dual slope DVMs. IEEE Transactions on Instrumentation \& Measurement, 52 , (5), 1588-1592.

[10] Cichy, A. (2013). Non-bridge circuit with double quasi-balancing for measurement of dielectric loss factor. IET Science Measurement \& Technology, 7 (5), 274-279.

[11] Roj, J. (2013). Neural approximation of empirical functions. Acta Physica Polonica A, 124 (3), 554-557.

[12] Hornik, K., Stinchcombe, M., White, H. (1989). Multilayer feedforward networks are universal approximators. Neural Networks, 2, 359-366.

[13] Hornik, K. (1991). Approximation capabilities of multilayer feedforward networks. Neural Networks, 4 (2), 251-257.

[14] Haykin, S. (2008). Neural Networks: A Comprehensive Foundation. Third Edition. Prentice Hall.

[15] Gupta, M., Homma, N., Jin, L. (2003) Static and Dynamic Neural Networks: From Fundamentals to Advanced Theory. John Wiley \& Sons.

[16] Beale, M.H., Hagan, M.T., Demuth, H.B. (2015). Neural Network Toolbox: User's Guide. MathWorks Inc.

[17] Hagan, M.T., Menhaj, M. (1994). Training feedforward networks with the Marquardt algorithm. IEEE Transactions on Neural Networks, 5 (6), 989993.

[18] Glowacz, A., Glowacz, A., Glowacz, Z. (2014). Recognition of monochrome thermal images of synchronous motor with the application of quadtree decomposition and backpropagation neural network. Maintenance and Reliability (Eksploatacja $i$ Niezawodnosc), 16 (1), 92-96.

[19] Roj, J. (2014). Estimation of the artificial neural network uncertainty used for measurand reconstruction in a sampling transducer. IET Science Measurement \& Technology, 8 (1), 23-29. 